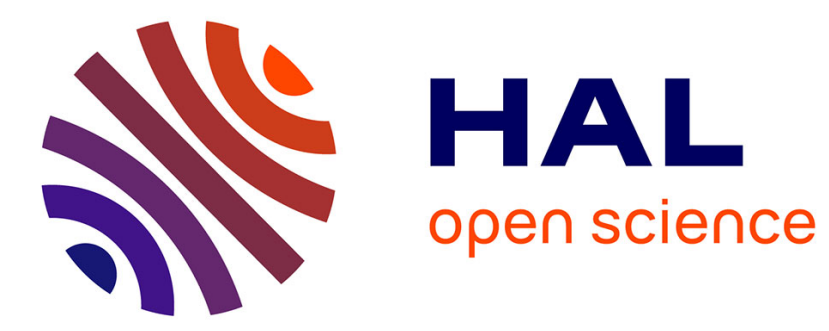

\title{
Impact des rejets de médicaments sur le milieu récepteur : résultats des projets Sipibel et Irmise
}

\author{
Laure Wiest, Robert Baudot, Emmanuelle Vulliet
}

\section{To cite this version:}

Laure Wiest, Robert Baudot, Emmanuelle Vulliet. Impact des rejets de médicaments sur le milieu récepteur: résultats des projets Sipibel et Irmise. Techniques Sciences Méthodes , 2016, 6, pp.12-21. $10.1051 / \mathrm{tsm} / 201606012$. hal-01355563

\section{HAL Id: hal-01355563 \\ https://hal.science/hal-01355563}

Submitted on 10 Sep 2020

HAL is a multi-disciplinary open access archive for the deposit and dissemination of scientific research documents, whether they are published or not. The documents may come from teaching and research institutions in France or abroad, or from public or private research centers.
L'archive ouverte pluridisciplinaire HAL, est destinée au dépôt et à la diffusion de documents scientifiques de niveau recherche, publiés ou non, émanant des établissements d'enseignement et de recherche français ou étrangers, des laboratoires publics ou privés. 
Impact des rejets de médicaments sur le milieu récepteur : résultats des projets SIPIBEL et IRMISE

\author{
L. WIEST ${ }^{1}$, R. BAUDOT ${ }^{1}$, E. VULLIET ${ }^{1}$
}

${ }^{1}$ Université de Lyon - Institut des Sciences Analytiques, UMR 5280 CNRS, Groupe TRACES, Université Lyon 1, ENS-Lyon -5 rue de la Doua -69100 Villeurbanne

Mots-clés : micropolluant, médicament, pharmaceutique, eau de surface, eau souterraine, rejet de station d'épuration

\title{
Introduction
}

La pollution de l'eau est une préoccupation majeure de notre société. La contamination avérée des eaux de surface a donné lieu à la directive cadre européenne sur l'eau 2000/60/CE du 23 octobre 2000 (DCE), dans laquelle est établie une liste de 41 substances à surveiller, comprenant des hydrocarbures, des métaux, des alkylphénols et des pesticides. L'acquisition de données sur ces composés a permis, en 2008 d'établir des Normes de Qualité Environnementales (NQE) (Directive $n^{\circ}$ 2008/105/CE). Mais la liste de la DCE ne comprend qu'une infime partie des micropolluants d'origine anthropique : parmi eux, d'autres pesticides, des plastifiants, des détergents et également les médicaments [LUO et al. 2014].

Les médicaments occupent une place à part parmi les micropolluants pour plusieurs raisons : d'une part, leurs quantités très faibles dans l'environnement (de l'ordre du $\mathrm{ng} / \mathrm{L}$ ) mais qui n'excluent pas un effet biologique, requièrent des techniques d'analyse de pointe ; d'autre part, si certains médicaments sont éliminés sous forme inchangée, la plupart sont métabolisés en un ou plusieurs métabolites plus ou moins actifs biologiquement [FATTA-KASSINOS et al. 2011]. Les principales sources de contamination sont connues : les rejets domestiques, les rejets associés à l'industrie pharmaceutique, les établissements de soin, les rejets associés aux activités vétérinaires et à la pisciculture et les apports provenant de médicaments non utilisés [BOXALL et al. 2012]. Leur élimination dans les stations d'épuration (STEP) a été très étudiée ces dernières années. Elle est très variable en fonction des médicaments : de 100\% pour le paracétamol, à des taux d'élimination nuls voire négatifs pour la carbamazépine [VERLICCHI et al. 2012, SOULIER et al. 2011]. Mais peu de travaux concernent également leur impact sur le milieu récepteur et encore moins sur les eaux souterraines, ce qui est pourtant indispensable à l'évaluation de l'exposition humaine [PETRIE et al. 2015].

Cet article est issu du travail mené dans le cadre des projets SIPIBEL (site pilote de Bellecombe) et IRMISE Arve aval [BRELOT et al. 2013], dont l'un des objectifs est d'étudier à l'échelle d'un territoire les transferts de médicaments, des sorties de STEP, jusqu'aux eaux souterraines destinées à la production d'eau potable, en passant par le milieu récepteur des rejets de STEP, c'est-à-dire, l'Arve. Plus précisément, 12 substances pharmaceutiques, appartenant à 7 classes différentes - analgésique (paracétamol, acide salicylique qui est un métabolite de l'aspirine), anti-inflammatoire (diclofénac, ibuprofène, kétoprofène), bétabloquant (aténolol, propranolol), antifongique (econazole), antiépileptique (carbamazépine), antibiotique (ciprofloxacine, sulfaméthoxazole) et hormone (17 $\alpha$ éthinylestradiol) - ont été suivis sur les observatoires SIPIBEL et IRMISE Arve-Aval. Les concentrations en micropolluants étant très variables et dépendantes des conditions climatiques [COQUERY et al. 
2011], un suivi sur deux ans a été réalisé avec un minimum de 9 campagnes, par site de prélèvement. Ces résultats permettent d'une part, de comparer les concentrations en médicaments de différents effluents traités, y compris celles d'un effluent provenant exclusivement d'un hôpital et d'autre part, d'étudier l'impact de ces rejets sur le milieu récepteur, à la fois au niveau des eaux de surface et souterraines.

\section{Matériel et méthodes}

\subsection{Sites d'étude et échantillonnage}

\subsubsection{Sites étudiés}

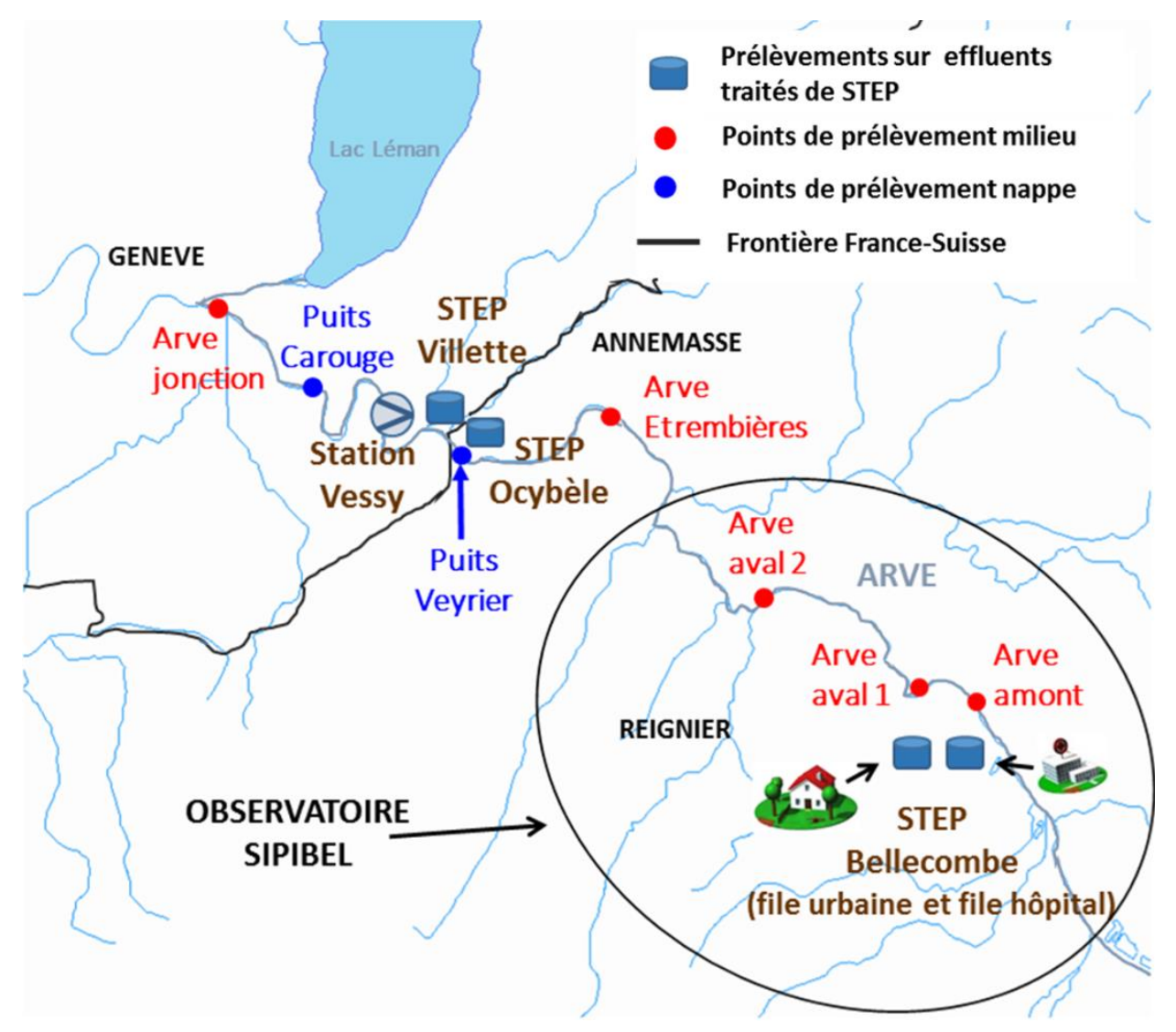

Figure 1. Sites de prélèvement (STEP, milieu et nappe) considérés dans cette étude

Le site SIPIBEL, ainsi que le territoire d'étude du projet IRMISE Arve Aval, dans lesquels s'est déroulée cette étude ont déjà fait l'objet d'une publication [BRELOT et al. 2013]. Les sites de prélèvement dont les résultats sont présentés dans cet article comprennent :

- Quatre eaux traitées (ET) de STEP : deux sur le site de Bellecombe (effluent urbain et effluent hospitalier) et deux des STEP Ocybèle (France) et Villette (Suisse). Les caractéristiques de ces STEP, capacité nominale, débit moyen et temps de résidence hydraulique, sont présentées dans le Tableau I. Ces STEP comprennent une étape de pré-traitement, une étape de décantation et un traitement biologique par boues activées pour les STEP de Bellecombe et Villette et par biofiltration pour Ocybèle.

- Cinq points de l'Arve : trois proches de la STEP de Bellecombe (un en amont de la STEP, un en aval immédiat et un en aval éloigné (environ $5 \mathrm{~km}$ )) et deux proches des STEP d'Ocybèle et Villette (un en 
amont et un en aval des deux STEP). Les moyennes des débits mesurés lors des campagnes de prélèvement sont indiquées dans le Tableau I.

- La station de Vessy, qui permet de réalimenter la nappe du Genevois au moyen d'eau prélevée dans l'Arve : a ici été analysée l'eau injectée dans la nappe ayant subi un traitement (dessablage, floculation, filtration et chloration).

- Deux puits de la nappe du Genevois (Veyrier et Carouge), exploités pour la production d'eau potable : cette eau souterraine n'a pas subi de chloration.

\begin{tabular}{lrrrc}
\cline { 2 - 5 } & Capacité & $\begin{array}{c}\text { Débit moyen } \\
\mathrm{m}^{3} / \text { jour }\end{array}$ & $\begin{array}{l}\text { Temps de } \\
\text { Résidence }\end{array}$ & $\begin{array}{c}\text { Traitement } \\
\text { secondaire }\end{array}$ \\
\hline Bellecombe Urbain & $26600 \mathrm{EH}$ & 5355 & 36 heures & Boues activées \\
\hline Bellecombe Hôpital & $5400 \mathrm{EH}$ & 146 & 240 heures & Boues activées \\
\hline Villette & $50000 \mathrm{EH}$ & 18081 & 12 heures & Boues activées \\
\hline Ocybèle & $125000 \mathrm{EH}$ & 17673 & 6 heures & Biofiltration \\
\hline Arve Bellecombe & - & 2794770 & - & - \\
\hline Arve Etrembières & - & 3805263 & - & - \\
\hline Arve Jonction & - & 3924435 & - & - \\
\hline
\end{tabular}

Tableau I. Caractéristiques des sites étudiés, capacité en équivalent-habitant (EH), débit moyen et temps de résidence hydraulique moyen, lors des campagnes de prélèvement, et type de traitement secondaire utilisé

\subsubsection{Campagnes et protocole de prélèvement}

Neuf campagnes ont été réalisées sur le territoire d'étude, sur 2 ans (janvier 2013 à janvier 2015). A chaque campagne, tous les points préalablement cités ont été échantillonnés le même jour. Afin d'éviter les phénomènes de dilution et d'assurer une bonne faisabilité des prélèvements, toutes les campagnes ont été effectuées en période de basses eaux de l'Arve avec comme critère supplémentaire, que le débit de la filière urbaine de la STEP de Bellecombe soit inférieur à 6000 $\mathrm{m} 3 /$ jour.

L'échantillonnage étant déterminant sur les résultats d'analyse, les recommandations du guide technique Aquaref [EYMERY et al. 2011] ont été suivies. Tous les échantillons de rejet de STEP et d'eaux de surface ont été prélevés sur $24 \mathrm{~h}$ (de $8 \mathrm{~h}$ à $8 \mathrm{~h}$ ), asservis au débit. Les échantillons d'eau ont été collectés à l'aide de préleveurs automatiques réfrigérés équipés de tuyaux en téflon et de flacons en verre : ASP Station 2000 (Endress hauser) pour les eaux de STEP et SIGMA 900SD pour les eaux de surface. Ils ont ensuite été homogénéisés et distribués mécaniquement dans des flacons, puis mis en glacière à $4^{\circ} \mathrm{C}$, livrés et traités dans les $24 \mathrm{~h}$ suivant le prélèvement (cf. section 1.2). En ce qui concerne les eaux de surface, 24 échantillons horaires ont été produits puis mélangés proportionnellement aux valeurs de débits de l'Arve fournies par EDF afin de reconstituer un échantillon $24 \mathrm{~h}$ représentatif. Les échantillons d'eau souterraine ont quant à eux été prélevés ponctuellement.

\subsection{Analyses chimiques}

La méthode utilisée pour analyser les substances pharmaceutiques comprend une extraction sur phase solide (SPE) suivie par une analyse par chromatographie liquide couplée à la spectrométrie de masse en tandem (LC-MS/MS). Cette stratégie a été élaborée à partir de méthodes publiées et 
validées sur des eaux de surface, effluents urbains [GROS et al. 2006] et effluents hospitaliers [GOMEZ et al. 2006].

\subsubsection{Extraction de la phase dissoute}

Après prélèvement et conservation à $4^{\circ} \mathrm{C}$, les échantillons d'eaux sont traités dans les $24 \mathrm{~h}$ suivant le prélèvement. Ils sont tout d'abord filtrés sur filtre en fibre de verre $0,7 \mu \mathrm{m}$, ce qui signifie que seule la contamination de la fraction dissoute est prise en compte dans cette étude. Chaque échantillon est ensuite dopé avec des standards internes (paracétamol-d3, sulfaméthoxazole-d4, ciprofloxacine-d8, miconazole-d5, kétoprofène-d4, diclofénac- $d 4$, ibuprofène-d3, acide salicylique-d6, aténolol-d7, propranolol-d7, 17 $\alpha$-éthinylestradiol-d4, carbamazépine-d10) avant l'étape d'extraction SPE. Cette dernière est réalisée sur un extracteur automatique de la marque Caliper nommé AutoTrace SPS Workstation, sur des cartouches SPE Oasis HLB (60 mg/3 mL). L'échantillon est percolé à $10 \mathrm{~mL} / \mathrm{min}$ sur les cartouches préalablement conditionnées avec $5 \mathrm{~mL}$ de méthanol, puis $5 \mathrm{~mL}$ d'eau ultra-pure. Le volume extrait dépend de la matrice analysée : sortie de STEP, $250 \mathrm{~mL}$ et eau de surface ou souterraine, $400 \mathrm{~mL}$. Les cartouches sont ensuite rincées avec $5 \mathrm{~mL}$ d'eau ultra-pure, séchées puis éluées avec deux fois $4 \mathrm{~mL}$ de méthanol. L'extrait obtenu est évaporé, repris dans $1 \mathrm{~mL}$ d'eau et congelé jusqu'à analyse.

\subsubsection{Analyse par chromatographie liquide couplée à la spectrométrie de masse}

L'appareillage utilisé pour l'analyse est de marque Agilent/ série 1100 pour la chromatographie liquide (LC) et ABSciex/ 3200 QTRAP pour la spectrométrie de masse en tandem (MS/MS). Les données sont acquises et retraitées grâce au logiciel Analyst (version 1.5.2). L'analyse est réalisée en phase inverse (gradient d'élution) sur une colonne Zorbax Eclipse Plus C18 1,8 $\mu \mathrm{m}(50 * 2,1) \mathrm{mm}$ (Agilent), chauffée à $60^{\circ} \mathrm{C}$. Le volume injecté est de $50 \mu \mathrm{L}$, la phase aqueuse utilisée consiste en de l'eau ultra-pure avec de l'acide acétique $(100 \mu \mathrm{L}$ dans $1 \mathrm{~L})$ et la phase organique du méthanol en mode positif, et un mélange méthanol/acétonitrile 50/50 en mode négatif. Paracétamol, aténolol, ciprofloxacine, sulfaméthoxazole, propranolol, carbamazépine, kétoprofène, econazole et diclofénac sont analysés en mode positif. Acide salicylique, ibuprofène, 17 $\alpha$-éthinylestradiol sont analysés en mode négatif. Chaque molécule est identifiée par son temps de rétention, par 2 transitions MRM (ion parent-> ion fils) et des rapports d'ions de l'ordre de $+/-30 \%$ par rapport à l'étalon. Chaque extrait est injecté 4 fois : deux fois en mode positif, et deux fois en mode négatif ; et les résultats présentés sont la moyenne des deux mesures dans chaque mode.

\subsubsection{Quantification et contrôle qualité}

La sensibilité obtenue de cette méthode est compatible avec l'analyse environnementale, les limites de quantification se situant entre 0,5 et 35 nanogrammes par litre (ng/L) pour les rejets de STEP et entre 0,1 et $10 \mathrm{ng} / \mathrm{L}$ pour les eaux de surface et souterraines. Afin de pallier aux effets de matrice, dus à la présence d'interférents dans l'extrait injecté, une méthode de quantification par calibration interne a été utilisée : chaque composé est quantifié par étalonnage interne, avec son propre standard interne (cf. section 1.2.1), seule méthode, à l'heure actuelle, qui permette une quantification fiable dans des matrices aussi complexes [HEWAVITHARANA 2011]. Enfin, des blancs analytiques et des contrôles qualité ont été effectués à chaque séquence d'analyses. Des campagnes de blanc de prélèvement ont également régulièrement été réalisées durant les deux années, avec de l'eau ultra-pure, de l'eau minérale et des échantillons réels, afin d'évaluer les phénomènes de 
contamination (qui pourraient conduire à surestimer le résultat) et d'adsorption de micropolluants dans le préleveur (sous-estimation du résultat).

\section{Résultats et discussions}

\subsection{Rejets de STEP (Hôpital,Urbain,Ocybèle (France) et Villette (Suisse))}

Sur les 12 molécules recherchées, 3 ont peu ou pas été quantifiées : I'acide salicylique, l'econazole et la $17 \alpha$-éthinylestradiol. Bien que récemment répertoriée dans les listes de substances à surveiller, l'éthinylestradiol n'a également pas été détectée dans plusieurs études [ZORITA et al. 2009, NAGARNAIK et al. 2010]. Ceci peut s'expliquer par le fait que lorsqu'elle est quantifiée, c'est à des concentrations, inférieures à la dizaine de $\mathrm{ng} / \mathrm{L}$ voire au $\mathrm{ng} / \mathrm{L}$, alors que les méthodes d'analyse multirésidus ne permettent pas de descendre à des seuils aussi bas. L'acide salicylique, très bien éliminé par les STEP [VERLICCHI et al. 2012], n'a également pas été retrouvé. Enfin, l'econazole, ayant un caractère plus hydrophobe que les autres substances pharmaceutiques recherchées dans cette étude, a une grande affinité pour la fraction particulaire [LACHASSAGNE 2014], qui n'a pas été analysée dans ces travaux.

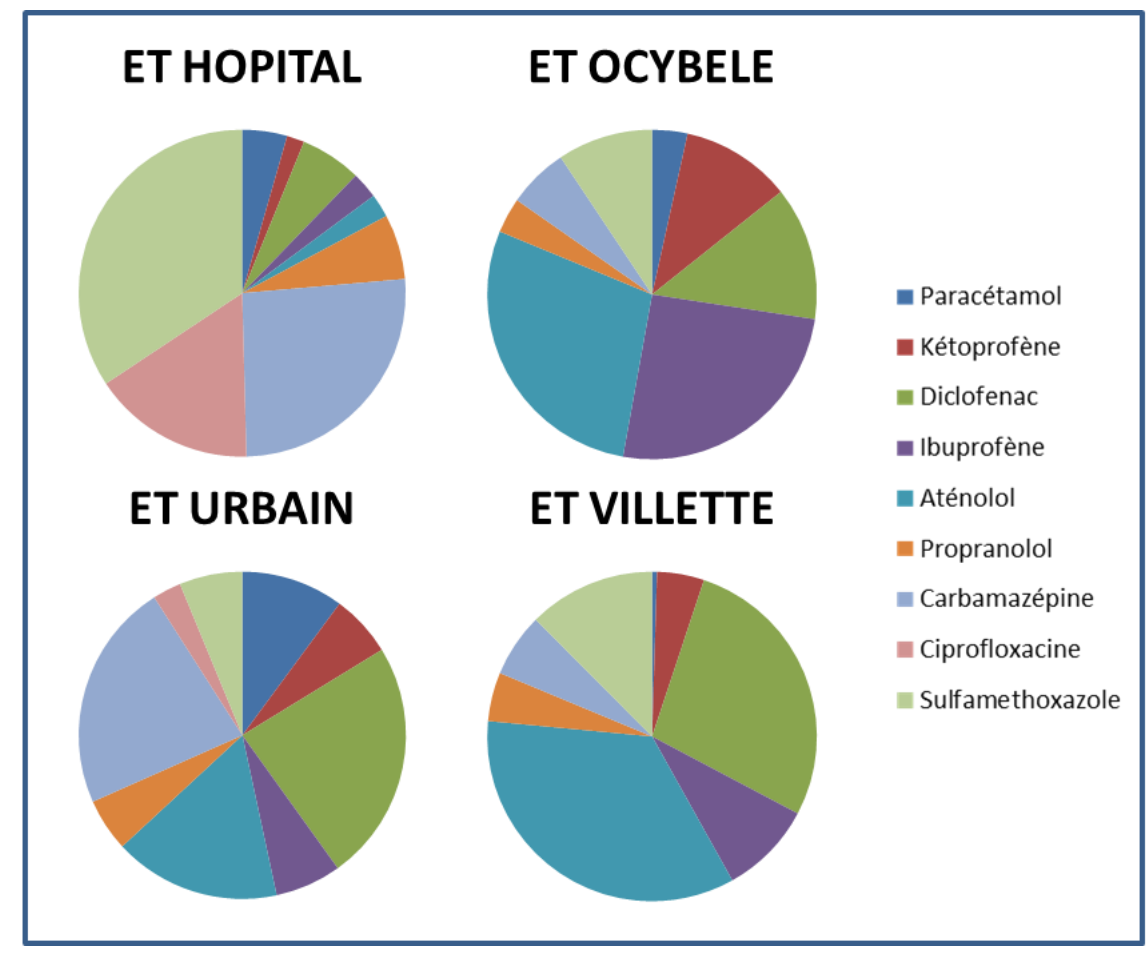

Figure 2. Contribution de chaque composé à la somme des substances analysées dans les eaux en sorties des stations d'épuration étudiées - Observatoire SIPIBEL-IRMISE (Janvier 2013 à Janvier 2015 ; 9 campagnes)

D'un point de vue qualitatif, le rejet hospitalier est très différent des trois rejets urbains : la Figure 2 présente la contribution de chaque composé à la somme des substances analysées. Cette figure met en évidence la spécificité de l'eau traitée hôpital, avec de fortes proportions en antibiotiques, ciprofloxacine et sulfaméthoxazole. Les eaux traitées urbaines ont des compositions assez semblables, avec la présence majoritaire de diclofénac et aténolol. Elles ont néanmoins quelques spécificités, avec une forte proportion en carbamazépine dans l'eau traitée de Bellecombe et une forte proportion en ibuprofène dans celle d'Ocybèle. 
D'un point de vue quantitatif, au cours des 9 campagnes, la somme des concentrations des 12 substances pharmaceutiques quantifiées dans les rejets de STEP a toujours été supérieure à $1 \mu \mathrm{g} / \mathrm{L}$.
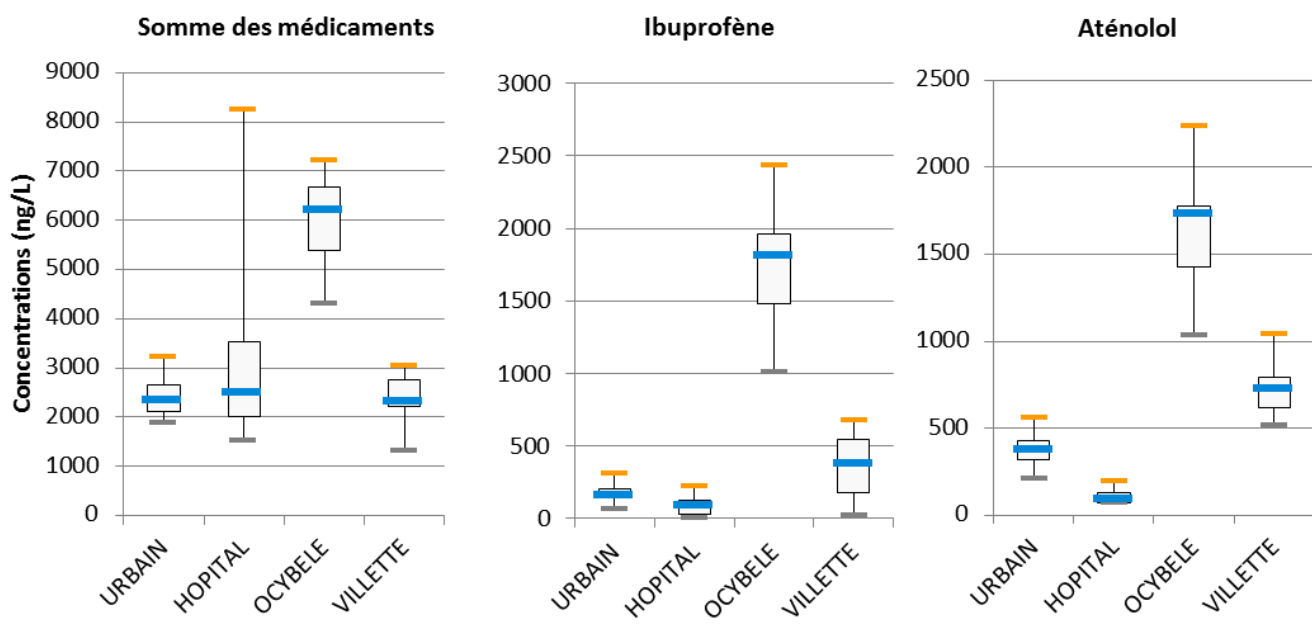

\section{- Maximum \\ 3ème quartile* \\ - Médiane}
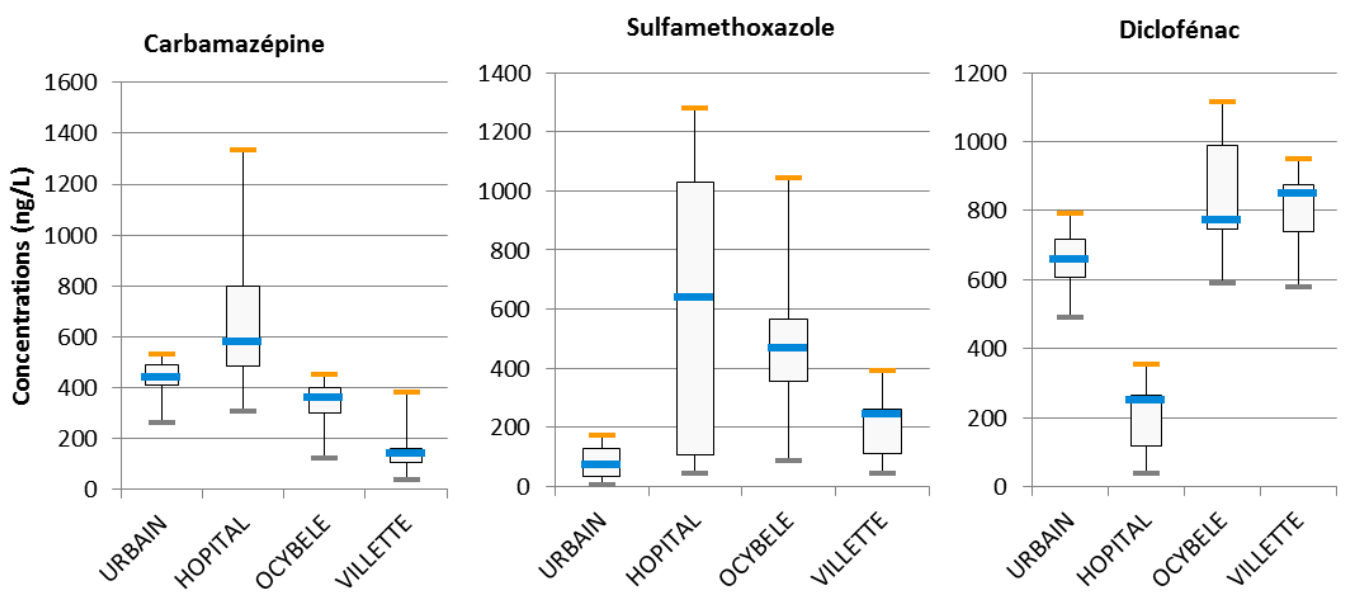

1er quartile*

- Minimum

Figure 3. Concentrations en $\mathrm{ng} / \mathrm{L}$ des rejets de stations d'épuration (STEP) (Janvier 2013 à Janvier 2015; 9 campagnes)

Les concentrations s'échelonnent entre la dizaine de $\mathrm{ng} / \mathrm{L}$ et le $\mu \mathrm{g} / \mathrm{L}$, ce qui est cohérent avec la littérature [VERLICCHI et al. 2012]. La Figure 3 présente la somme des concentrations des substances recherchées, ainsi que les concentrations des 5 composés quantifiés aux valeurs les plus élevées, sous forme de " boîtes à moustaches ". Cette forme de présentation permet de montrer la variabilité des concentrations avec de bas en haut, le minimum, le $1^{\text {er }}$ quartile, la médiane, le $3^{\text {ème }}$ quartile et le maximum. Une forte variabilité des concentrations, y compris celles de l'eau traitée issue de I'hôpital, peut être observée, ce qui est en accord avec la littérature [SOULIER et al. 2011]. La Figure 3 montre également des différences significatives en termes de concentrations de substances pharmaceutiques des 4 eaux traitées analysées. Ces différences peuvent provenir à la fois des concentrations en entrées de STEP, de la nature de l'eau brute et des filières de traitement des STEP. Contrairement à ce qui pourrait être attendu, les concentrations les plus fortes en médicaments ne sont pas celles provenant du rejet hospitalier, hormis pour l'antibiotique, sulfamethoxazole et l'anti-épileptique, carbamazépine. Par exemple, pour le diclofénac, les concentrations dans les rejets domestiques sont toutes significativement supérieures au rejet hospitalier. Ceci peut s'expliquer par la différence de temps de résidence hydraulique entre les filières urbaines et hôpital (cf. Tableau I) : en effet, un temps de résidence hydraulique plus long, 
permet d'éliminer plus efficacement certaines substances pharmaceutiques, comme le diclofénac [GUERRA et al. 2014].

En ce qui concerne la somme des concentrations en substances pharmaceutiques, le rejet de la STEP Ocybèle est celui qui présente les concentrations les plus élevées, en particulier en ibuprofène et en aténolol. Deux raisons peuvent expliquer cette différence : des concentrations plus élevées en entrée de STEP, et un temps de résidence hydraulique plus court au niveau de la STEP Ocybèle, à savoir 6 heures, contre 12 heures et 1,5 jours sur les STEP Villette et Bellecombe, respectivement. Cependant, les concentrations maximales retrouvées, à savoir $2,4 \mu \mathrm{g} / \mathrm{L}$ en ibuprofène et $2,2 \mu \mathrm{g} / \mathrm{L}$ en aténolol restent bien en dessous des concentrations maximum reportées dans la littérature, qui s'élèvent à 7,6 et $55 \mu \mathrm{g} / \mathrm{L}$, respectivement [LUO et al., 2014].

Enfin, le flux total en médicaments rejeté par chaque STEP est bien corrélé à leur dimension : en moyenne $7 \mathrm{~g} / \mathrm{j}$ pour Bellecombe contre 38 et $102 \mathrm{~g} / \mathrm{j}$ pour Villette et Ocybèle respectivement. Le flux moyen du rejet hospitalier s'élève quant à lui à $0,4 \mathrm{~g} / \mathrm{j}$, ce qui représente finalement une contribution faible au regard des flux apportés par les rejets domestiques.

\subsection{Impact sur le milieu récepteur : concentrations dans l'Arve}
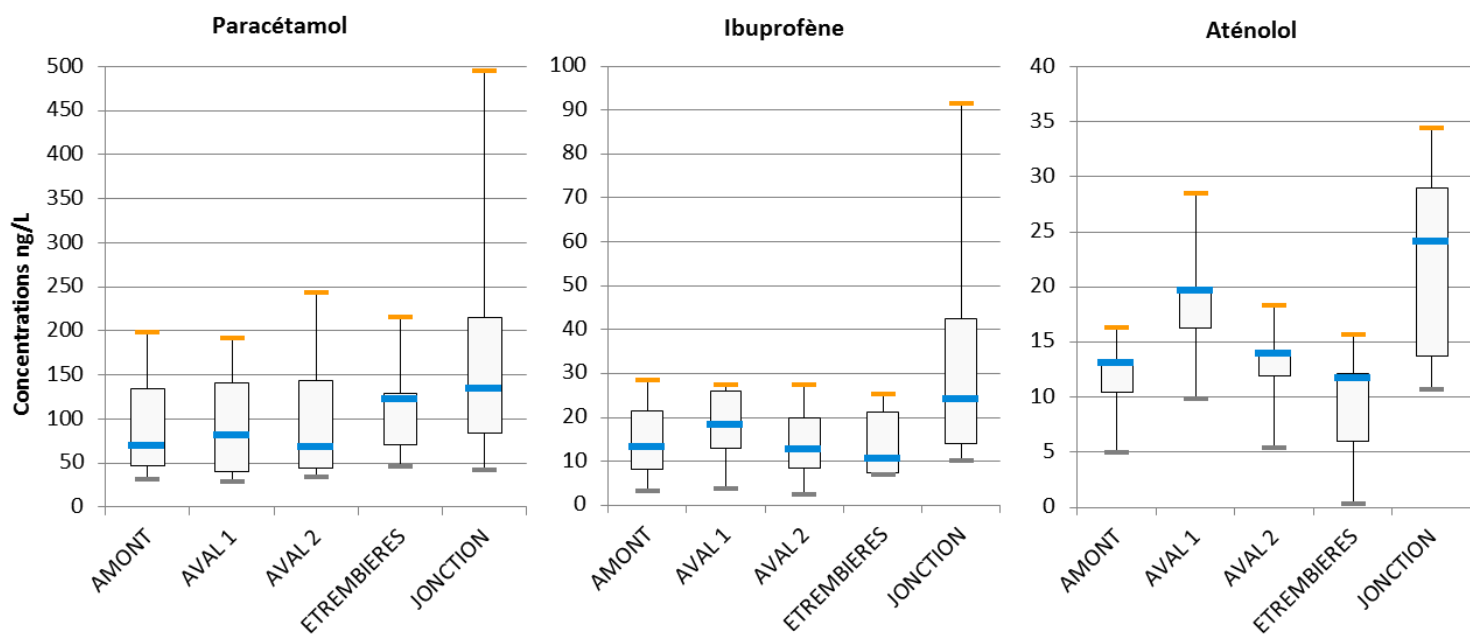

- Maximum
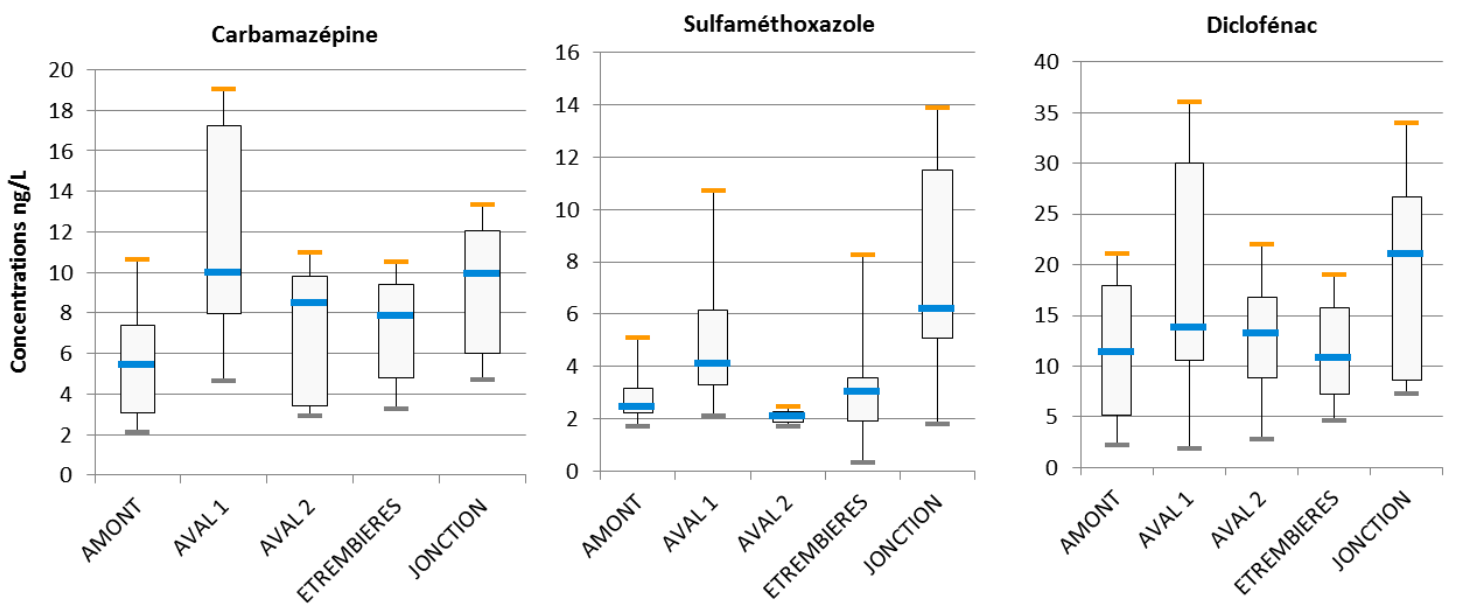

3ème quartile*

-Médiane 1 er quartile*

- Minimum

Figure 4. Concentrations en ng/L dans l'Arve (Janvier 2013 à Janvier 2015; 9 campagnes) 
Les 9 substances pharmaceutiques quantifiées dans les rejets de STEP ont été détectées dans l'Arve, dont 8 à des fréquences supérieures à $50 \%$ : le paracétamol, les anti-inflammatoires (ibuprofène, kétoprofène et diclofénac), les bétabloquants (aténolol et propranolol), la carbamazépine et l'antibiotique sulfaméthoxazole. Les concentrations observées sont de l'ordre du ng/L pour le kétoprofène et le propranolol, et jusqu'à la centaine de $\mathrm{ng} / \mathrm{L}$ pour le paracétamol. Les valeurs retrouvées pour les 6 substances pharmaceutiques quantifiées aux concentrations les plus élevées sont représentées dans la Figure 4. Ces concentrations sont du même ordre de grandeur voire inférieures aux concentrations médianes des eaux de surface reportées dans le monde [HUGHES et al. 2013]. Elles sont également en accord avec l'étude de 2010 [ORTELLI et al. 2011] dans laquelle des pesticides et médicaments avaient été recherchés sur les points Arve Etrembières et Jonction. La carbamazépine, le kétoprofène et le sulfamethoxazole n'avaient pas été quantifiés, ce qui peut s'expliquer par des limites de quantification supérieures ou égales à $10 \mathrm{ng} / \mathrm{L}$.

Toutes les substances pharmaceutiques, sauf le paracétamol, montrent globalement la même évolution le long de l'Arve. Tout d'abord, une hausse en aval immédiat de la STEP de Bellecombe (Aval 1), liée au panache du rejet de la STEP, est observée. Cette concentration se dissipe en aval éloigné (Aval 2), sûrement en raison du facteur de dilution élevé entre la STEP et l'Arve qui est en moyenne de 400 en période d'étiage, ou de phénomènes d'adsorption et de photobiodégradation. Les concentrations entre Aval 2 et Arve Etrembières sont très proches, ce qui suggéré qu'il n'y a pas $d$ 'apport supplémentaire entre ces deux points. Enfin, on observe une hausse significative des concentrations entre l'amont (Arve Etrembières) et l'aval (Arve jonction) des deux STEP Ocybèle et Villette. La somme des flux de résidus pharmaceutiques rejetés par ces deux STEP et du flux au point Arve Etrembières concorde avec le flux au point Arve jonction : on peut donc supposer qu'il n'y a pas d'autre apport majeur en résidus de substances pharmaceutiques et que cette hausse provient bien du rejet des deux STEP.

L'évolution du paracétamol est très différente. D'une part, celui-ci est déjà très présent en amont de la STEP de Bellecombe, avec une concentration médiane à $68 \mathrm{ng} / \mathrm{L}$. D'autre part, on n'observe pas de hausse de concentration liée à l'apport de rejets de STEP, mais une hausse entre Aval 2 et Arve Etrembières, ce qui suggère qu'il y aurait une autre source de paracétamol entre ces deux points. D'après cette étude, les sources de paracétamol seraient donc plus diffuses que celles des autres substances pharmaceutiques.

Ces observations sont en accord avec d'autres études de screening de substances pharmaceutiques sur le territoire français. Les mêmes molécules ont été quantifiées dans le bassin versant de la Seine [MOREAU-GUIGNON et al. 2010], dans le bassin Loire-Bretagne [AMALRIC et al. 2011], et par l'agence de l'eau Artois-Picardie (AEAP) [AEAP 2010], ce qui montre l'ubiquité de ces substances dans les cours d'eaux, sûrement liée à leur forte consommation. Les ordres de grandeurs de concentrations sont également cohérents, sauf dans le bassin Artois-Picardie, où des concentrations de l'ordre de la centaine de $\mathrm{ng} / \mathrm{L}$ avaient été retrouvées, ce qui peut s'expliquer par la forte densité de population de cette région. Moreau-Guignon et al. [MOREAU-GUIGNON et al. 2010] ont également étudié le profil longitudinale de la Seine et montré une forte augmentation de concentrations en antibiotiques entre l'amont et l'aval de la STEP de Seine Aval, notamment en sulfaméthoxazole. 


\subsection{Eaux souterraines}

Sur les 12 substances pharmaceutiques recherchées, aucune n'a été quantifiée dans le puits Veyrier, une seule sur le puits Carouge, le sulfaméthoxazole, et trois sur la station Vessy, l'ibuprofène et la carbamazépine à des concentrations entre 0,5 et $3 \mathrm{ng} / \mathrm{L}$ et de manière très ponctuelle, et l'antibiotique, sulfaméthoxazole, sur l'ensemble des campagnes. Les concentrations en sulfaméthoxazole sont très faibles et répétables puisqu'elles s'échelonnent entre 1,7 et $2,6 \mathrm{ng} / \mathrm{L}$ sur le puits Carouge et 1,9 et 2,8 ng/L sur la station Vessy.

La présence de sulfaméthoxazole dans l'eau du puits Carouge peut s'expliquer soit par une source diffuse telle que l'utilisation en agriculture en tant que médicament vétérinaire, soit par des interactions entre l'eau souterraine et l'eau de surface. La présence de ce composé dans les eaux souterraines a déjà été reportée dans la littérature. Barnes et al. , en 2008 [BARNES et al. 2008], ont réalisé un très large screening de contaminants dans les eaux souterraines des Etats-Unis. Le sulfaméthoxazole faisait partie des composés les plus détectés ( $23 \%$ des échantillons), avec des concentrations entre $20 \mathrm{ng} / \mathrm{L}$ et $1 \mu \mathrm{g} / \mathrm{L}$. L'ibuprofène avait également été détecté mais seulement sur $2 \%$ des échantillons. Banzhaf et al. [BANZHAF et al. 2013] ont également étudié le transfert de substances pharmaceutiques entre une eau de surface et une eau souterraine, démontrant le caractère particulièrement mobile de trois d'entre elles : la carbamazépine et deux antibiotiques, sulfaméthazine et sulfaméthoxazole.

Le cas de la station Vessy est différent : celle-ci étant une station de réalimentation de la nappe au moyen d'eau prélevée dans l'Arve, les substances retrouvées proviennent certainement de la contamination initiale de l'Arve. Cependant, l'eau analysée ayant été traitée, ces résultats montrent la résistance du sulfaméthoxazole au procédé de traitement spécifique appliqué à la station de réalimentation de Vessy. La présence de sulfaméthoxazole dans l'eau potable a déjà été publiée dans la littérature [CAPDEVILLE and BUDZINSKI, 2011], avec des concentrations comprises entre 0,3 et 3,4 $\mathrm{ng} / \mathrm{L}$.

\section{Conclusions et perspectives}

Cette étude a permis de suivre douze substances pharmaceutiques fortement consommées, sur l'ensemble d'un territoire, des rejets de stations d'épuration aux eaux souterraines. Huit molécules ont été quantifiées à la fois dans les eaux traitées de STEP et les eaux de surface, à des teneurs entre 100 et $2000 \mathrm{ng} / \mathrm{L}$ dans les rejets, et de 1 à $200 \mathrm{ng} / \mathrm{L}$ dans les eaux de l'Arve, mettant en évidence l'effet de dissipation dans les eaux de surface. L'analyse spécifique du rejet provenant d'un hôpital n'a révélé qu'une faible contribution de celui-ci au flux total de médicaments. Enfin, une seule molécule a été retrouvée à l'état de traces dans les eaux souterraines : il s'agit de l'antibiotique, sulfaméthoxazole. Compte-tenu de la variabilité des concentrations, ce travail met également en exergue la nécessité de répéter les prélèvements sur un même site, afin d’obtenir des données pertinentes et représentatives d'un milieu.

D'un point de vue environnemental, cette étude montre la présence simultanée de diverses familles de médicaments dans les eaux de surface, alors que la liste considérée ne représente qu'une faible part des 3000 substances pharmaceutiques actuellement consommées. Or, très peu de données existent à l'heure actuelle, sur l'effet de ce mélange, appelé communément « effet cocktail » sur les organismes vivants. De plus, seule la fraction dissoute a été prise en compte, alors que certaines substances sont connues pour leur affinité avec la phase particulaire, telles que la ciprofloxacine ou l'econazole (cf. article de LACHASSAGNE et al. de ce dossier). Enfin, le sulfaméthoxazole ayant été 
quantifié dans les trois différents types d'eaux, y compris celles destinées à la production d'eau potable, son utilisation en tant que traceur de contamination anthropique pourrait s'avérer pertinente, comme déjà mentionné dans la littérature [BANZHAF et al., 2013, BRUCHET et al., 2015].

\section{Remerciements}

Les auteurs remercient les membres et partenaires de SIPIBEL - Site Pilote de Bellecombe sur les effluents hospitaliers et stations d'épuration - et du projet Interreg franco-suisse IRMISE Arve aval pour leurs contributions et en particulier, le Syndicat des eaux des Rocailles et de Bellecombe, Annemasse Les Voirons Agglomération, la Communauté de Communes du Genevois, l'Etat de Genève, les Services Industriels de Genève et le GRAIE pour la réalisation des prélèvements et la mise à disposition des données. Ils remercient également l'Agence de l'eau Rhône-Méditerranée Corse, la Région Rhône-Alpes, l'Union Européenne, les ministères en charge de la santé et de l'environnement, le conseil général de Haute-Savoie et l'ARS Rhône-Alpes pour leur soutien. Enfin, ils remercient EDF pour la transmission des données de débits de l'Arve.

\section{Bibliographie}

AEAP (2010) : Les médicaments dans les cours d'eau du bassin Artois-Picardie: résultats de la campagne exploratoire 2010. Rapport AEAP/DRM/SEM, EA/CH/JP - déc. 2010.

AMALRIC L., TOGOLA A., LOPEZ B. (2011) : Suivi des résidus de substances pharmaceutiques dans les systèmes aquatiques du bassin Loire-Bretagne. Rapport final. BRGM/RP-59371-FR.

BANZHAF S., KREIN A., SCHEYTT T. (2013) : « Using selected pharmaceutical compounds as indicators for surface water and groundwater interaction in the hyporheic zone of a low permeability riverbank ». Hydrological Processes; 27 : 2892-2902.

BARNES K. K., KOLPIN D. W., FURLONG E. T., ZAUGG S. D., MEYER M. T., BARBER L. B. (2008) : « A national reconnaissance of pharmaceuticals and other organic wastewater contaminants in the United States - I) Groundwater ». Science of the Total Environment ; 402 : 192-200.

BOXALL A. B. A., RUDD M. A., BROOKS B. W., CALDWELL D. J., CHOI K., HICKMANN S., et al. (2012) : « Pharmaceuticals and Personal Care Products in the Environment: What Are the Big Questions? ". Environmental Health Perspectives; 120 : 1221-1229.

BRELOT E., LECOMTE V., PATOIS, L. (2013) : « Le site pilote de Bellecombe (Sipibel) sur les effluents hospitaliers et stations d'épuration urbaines : premiers résultats du suivi ». TSM ; $12: 85-101$. BRUCHET A., MARTIN S., COQUERY M. (2015) : «Indicateurs chimiques d'efficacité de traitement et d'influence des rejets de stations d'épuration sur le milieu récepteur ». TSM ; 3 : 15-30.

CAPDEVILLE M. J., AND BUDZINSKI H. (2011) : « Trace-level analysis of organic contaminants in drinking waters and groundwaters ». Trends in Analytical Chemistry ; 30 : 586-606.

COQUERY M., POMIES M., MARTIN-RUEL S., BUDZINSKI H., MIÈGE C., ESPERENZA M., CHOUBERT J.M. (2011) : "Mesurer les micropolluants dans les eaux usées brutes et traitées : protocoles et résultats pour l'analyse des concentrations et des flux ". TSM ; 1/2:25-43.

EYMERY F., CHOUBERT J.-M., LEPOT B., GASPERI J., LACHENAL J., COQUERY M. (2011). Guide technique opérationnel: Pratiques d'échantillonnage et de conditionnement en vue de la recherche de micropolluants prioritaires et émergents en assainissement collectif et industriel. Première version. Irstea/Cemagref. 
FATTA-KASSINOS D., MERIC S., NIKOLAOU, A. (2011) : « Pharmaceutical residues in environmental waters and wastewater: current state of knowledge and future research ». Analytical and Bioanalytical Chemistry; 399 : 251-275.

GOMEZ M. J., PETROVIC M., FERNANDEZ-ALBA A. R., BARCELO D. (2006) : « Determination of pharmaceuticals of various therapeutic classes by solid-phase extraction and liquid chromatographytandem mass spectrometry analysis in hospital effluent wastewaters ". Journal of Chromatography A; 1114 : 224-233.

GROS M., PETROVIC M., BARCELO D. (2006) : « Development of a multi-residue analytical methodology based on liquid chromatography-tandem mass spectrometry (LC-MS/MS) for screening and trace level determination of pharmaceuticals in surface and wastewaters ». Talanta; 70 : 678690.

GUERRA P., KIM M., SHAH A., ALAEE M., SMYTH S. A. (2014) : « Occurrence and fate of antibiotic, analgesic/anti-inflammatory, and antifungal compounds in five wastewater treatment processes ". Science of the Total Environment; $473: 235-243$.

HEWAVITHARANA A. K. (2011) : " Matrix matching in liquid chromatography-mass spectrometry with stable isotope labelled internal standards-Is it necessary? ». Journal of Chromatography A; 1218 : 359-361.

HUGHES S. R., KAY P., BROWN L. E. (2013) : " Global Synthesis and Critical Evaluation of Pharmaceutical Data Sets Collected from River Systems ». Environmental Science \& Technology ; 47 : 661-677.

LACHASSAGNE D. (2014) : Devenir de micropolluants présents dans les boues d'épuration, du traitement à l'épandage agricole : Application aux micropolluants métalliques $(\mathrm{Cd}, \mathrm{Cu})$ et organiques (médicaments) issus du traitement biologique conventionnel d'effluents urbains ou hospitaliers. Thèse de doctorat, Université de Limoges.

LUO Y., GUO W., NGO H. H., LONG DUC N., HAI F. I., ZHANG J., et al. (2014) : « A review on the occurrence of micropollutants in the aquatic environment and their fate and removal during wastewater treatment ». Science of the Total Environment; 473 : 619-641.

MOREAU-GUIGON E., TAMTAM F., QUOC DINH T., EURIN J., LABADIE P., ALLIOT F., et al. (2010) : Sources et devenir des médicaments dans le bassin versant de la Seine. Rapport de synthèse PIRENSeine 2007-2010.

NAGARNAIK P. M., MILLS M. A., BOULANGER B. (2010) : " Concentrations and mass loadings of hormones, alkylphenols, and alkylphenol ethoxylates in healthcare facility wastewaters ».

Chemosphere; 78 : 1056-1062.

ORTELLI D., EDDER P., RAPIN F., RAMSEIER S. (2011). Métaux et micropolluants organiques dans les rivières et les eaux du Léman, campagne 2010. Rapp. Comm. int. prot. eaux Léman contre pollut., Campagne 2010.

PETRIE B., BARDEN R., KASPRZYK-HORDERN B. (2015) : « A review on emerging contaminants in wastewaters and the environment: Current knowledge, understudied areas and recommendations for future monitoring ». Water Research; 72 : 3-27.

SOULIER C., GABET V., LARDY S., LEMENACH K., PARDON P., ESPERANZA M., et al. (2011) : « Zoom sur les substances pharmaceutiques : présence, partition, devenir en station d'épuration ». TSM ; 1/2 : 65-77.

VERLICCHI P., AL AUKIDY M., AND ZAMBELLO E. (2012) : « Occurrence of pharmaceutical compounds in urban wastewater: Removal, mass load and environmental risk after a secondary treatment-A review ". Science of the Total Environment; 429 : 123-155. 
ZORITA S., MARTENSSON L., AND MATHIASSON L. (2009) : " Occurrence and removal of pharmaceuticals in a municipal sewage treatment system in the south of Sweden ». Science of the Total Environment; 407 : 2760-2770. 
Impact des rejets de médicaments sur le milieu récepteur : résultats des projets SIPIBEL et IRMISE Résumé

La contamination des milieux aquatiques par des micropolluants anthropiques est aujourd'hui avérée. Parmi eux, figurent des pesticides, des plastifiants, des détergents et les médicaments, qui occupent une place particulière de par leur très faible concentration dans l'environnement et la présence potentielle de métabolites. Bien que leur comportement dans les stations d'épuration (STEP) soit bien connu, beaucoup moins d'études se consacrent à leur devenir dans le milieu récepteur. Dans le cadre des projets SIPIBEL et IRMISE, cet article présente le résultat d'un suivi régulier de deux ans, de douze substances pharmaceutiques sur la partie aval du bassin versant de la rivière Arve, des rejets de station d'épuration y compris celui provenant d'un hôpital, aux eaux souterraines destinées à la production d'eau potable. L'utilisation de techniques d'analyse de pointe a permis d'atteindre des limites de quantification inférieures à la dizaine de $\mathrm{ng} / \mathrm{L}$, compatibles avec la recherche d'ultra-traces. Huit molécules, quantifiées dans les rejets de STEP à des teneurs de l'ordre $\mathrm{du} \mu \mathrm{g} / \mathrm{L}$, ont également été détectées dans le milieu récepteur, c'est-à-dire l'Arve, à des concentrations entre le $\mathrm{ng} / \mathrm{L}$ et la centaine de $\mathrm{ng} / \mathrm{L}$ (paracétamol). Bien que qualitativement différent des autres rejets de par sa forte proportion en antibiotiques, l'apport en médicaments du rejet hospitalier en termes de flux apparait faible comparé à celui des rejets domestiques. Trois substances, parmi les huit, ont également été quantifiées dans les eaux souterraines, notamment le sulfaméthoxazole, à des teneurs de l'ordre de $2 \mathrm{ng} / \mathrm{L}$. Ce dernier s'avère particulièrement résistant aux traitements, y compris à ceux réalisés dans le cadre de la production d'eau potable et pourrait donc se révéler comme un bon traceur de pollution anthropique dans l'environnement.

\section{Impact of pharmaceuticals discharges on the receiving environment: SIPIBEL and IRMISE projects results}

Abstract

Chemical water pollution is more and more studied and documented. Among these pollutants, drugs are special contaminants, due their very low concentrations in the environment and the potential presence of metabolites. Although their behavior in wastewater treatment plants (WWTP) is well known, many fewer studies are devoted to their fate in the receiving environment. Within the framework of the SIPIBEL and IRMISE projects, this article presents the results of a two years regular monitoring of twelve pharmaceutical residues, from treatment plant discharges, including one from a hospital, to groundwater intended for drinking water production. Thanks to the use of advanced analytical techniques, limits of quantification below $10 \mathrm{ng} / \mathrm{L}$, consistent with ultra-traces detection, were achieved. Eight molecules, quantified in WWTP discharges at $\mu \mathrm{g} / \mathrm{L}$ levels, were also detected in the receiving environment, which is the Arve River, in concentrations between the $\mathrm{ng} / \mathrm{L}$ and $100 \mathrm{ng} / \mathrm{L}$ for acetaminophen. Significant qualitative differences were observed between the hospital treatment discharge and domestic treated waters, due to its high proportion of antibiotics. But, in terms of mass flows, the hospital contribution appeared relatively low compared to domestic discharges. Three substances, among the eight, were also quantified in groundwater, particularly sulfamethoxazole, at the order of $2 \mathrm{ng} / \mathrm{L}$. This substance is very resistant to water treatments, including those made for drinking water production and therefore may be a good anthropogenic indicator. 